CIC. Cuadernos de Información y Comunicación ISSN: $1135-7791$

http://dx.doi.org/10.5209/CIYC.60688

\title{
La respuesta comunicativa de las ONGD andaluzas con la campaña '20 años del 0,7' en el contexto de crisis de la cooperación internacional
}

\author{
Óscar Toro Peña; Paloma Contreras Pulido; Ángel Hernando Gómez ${ }^{1}$
}

Recibido: 11 de febrero de 2018 / Aceptado: 11 de marzo de 2018

Resumen. El artículo analiza la respuesta comunicativa de la Coordinadora Andaluza de ONGD (CAONGD) con la campaña conmemorativa '20 años del 0,7' en el contexto de crisis de la cooperación internacional al desarrollo (2008-2015). Los resultados, tras la entrevista a responsables de la campaña y la celebración de un grupo de discusión, evidencian tres escenarios: el primero, el fracaso inicial de una estrategia convencional centrada en denunciar los efectos de los recortes en cooperación que no conectan con la ciudadanía; el segundo, tras un giro estratégico, apelando a la memoria colectiva y al recuerdo de las acampadas estatales que reivindicaron que se destinase el $0,7 \%$ del PIB a la cooperación al desarrollo; y el tercero, demostrando que el espacio comunicativo natural de las entidades es el que se articula desde los planteamientos de la comunicación para el cambio social.

Palabras clave: Cooperación internacional al desarrollo; ONGD; comunicación; conmemoración; coordinadora.

\section{[en] Communicative response of the andalusian ONGD with the campaign ' 20 years of the ' 0,7 ' in the context of crisis of the international cooperation}

\begin{abstract}
The article discusses the communicative response of the Andalusian Coordinator of NGO (CAONGD) with the commemorative campaing '20 year of $0,7^{\prime}$ ' in the context of the crisis of international cooperation for development (2008-2015). The results, after the interview responsible for the campaign and the celebration of a discussion group, show three scenarios: the first, the failure of an initial conventional approach focused report the effects of cuts in cooperation not connecting with citizens; the second, after a strategic shift, appealing to the collective memory and the memory of the State camps which claimed the $0.7 \%$ of GDP to development cooperation; and the third showing that natural communicative space of social entities which is articulated from the approaches of the communication for social change.
\end{abstract}

Keywords: International development cooperation; NGO; comunication; completed; coordinator.

Sumario. 1. Introducción. 2. Metodología. 3. Resultados. 4. Conclusiones. 5. Referencias bibliográficas.

Cómo citar: Toro Peña, Ó.; Contreras Pulido, P.; Hernando Gómez, Á. (2018). La respuesta comunicativa de las ONGD andaluzas con la campaña '20 años del 0,7' en el contexto de crisis de la cooperación internacional, en CIC. Cuadernos de Información y Comunicación 23, 201-213.

$1 \quad$ Universidad de Huelva 


\section{Introducción}

La cooperación internacional con sello español ha vivido, con la crisis financiera internacional, desde el año 2008 hasta el 2015, el periodo de mayor recorte de su historia. La Ayuda Oficial al Desarrollo (AOD) cae un $70 \%$, generando una pérdida del peso de España en la política exterior y un incumplimiento de sus acuerdos nacionales e internacionales. La cooperación ha sido durante años uno de los elementos más importantes de la política internacional (Sotillo, 2014). Reflejo de la solidaridad de la sociedad española, contribuyendo al desarrollo de países y pueblos empobrecidos, como instrumento de las relaciones exteriores y del compromiso del Gobierno a favor del multilateralismo. De ahí que tras la crisis no sólo ha habido un descenso de la ayuda, sino que España ha perdido solvencia en la acción exterior y ha debilitado su papel en el mundo. Además ha originado un retroceso, sin precedentes, en el sector de la cooperación internacional al desarrollo como lo evidencian los informes sobre las entidades del sector de la Coordinadora de Organizaciones de Cooperación para el desarrollo de España (CONGDE) de los años, 2009, 2011, 2012 y 2014.

Si se pone el foco en el año 2015 (último año de los recortes) los Presupuestos Generales del Estado destinan un 0,16\% de la Renta Nacional Bruta (RNB) a la AOD. O lo que es lo mismo, 1.813 millones de euros. En este mismo año Andalucía concede un $0,14 \%$ de su presupuesto, lo que significa 50.438 .345 euros. En esta partida se incluyen: la aportación a la Agencia Andaluza de Cooperación Internacional al Desarrollo (AACID), a las fundaciones públicas Tres Culturas y Barenboim, la ayuda multilateral (es decir, la contribución a organismos internacionales) y las partidas destinadas al Fondo Andaluz para la Promoción al Desarrollo (FAPRODE).

En este contexto Ignacio Martínez y Pablo Martínez, de la Plataforma 2015 y más, resumen lo que ha venido siendo la estrategia española en el campo de la cooperación internacional antes de la crisis, y que ellos han denominado el discurso cosmopolita, basado "en tres pilares: la adopción de una doctrina para posicionar a España en la agenda global de desarrollo, el aumento de la ayuda oficial al desarrollo (AOD) y el refuerzo de las estructuras para gestionar la AOD" (Martínez \& Martínez, 2010:45). Un discurso y unas políticas, en opinión de los autores, que tras los recortes han quedado seriamente debilitados. Y que según Gonzalo Fanjul, director del área de análisis de políticas de ISGlobal, en el artículo publicado en el especial de Planeta Futuro ${ }^{2}$ de El País (15/12/2015) sobre la cooperación española, se traduce en una derrota en tres frentes: presupuestario, político e institucional.

Si se observan los datos que ofrece el informe La Realidad de la Ayuda ${ }^{3}$ (2015) de la ONG Intermón Oxfam al comparar la aportación de fondos públicos en este ámbito de las diferentes comunidades autónomas (como actores públicos de desarro1lo) se evidencian unos comportamientos dispares de las administraciones regionales. A continuación, a modo de muestra, se destacan de forma comparativa las aportaciones de algunas comunidades tomando con referencia los años de principio y final del periodo de la crisis en España, es decir el 2008 y el 2015.

2 Planeta Futuro es la sección especializada en información al desarrollo que publica el diario el País desde enero del 2014 con el patrocinio de la Fundación Bill \& Melinda Gates.

3 La Realidad de la Ayuda es un informe que viene publicando anualmente desde el año 1994 Intermón Oxfam para evaluar de forma independiente la AOD y las políticas públicas en materia de cooperación internacional 
Tabla 1. Posición de comunidades autónomas con el cumplimiento del 0,7\%.

\begin{tabular}{|l|c|c|}
\hline \multicolumn{1}{|c|}{ Comunidad } & AOD 2008 & AOD2015 \\
\hline Navarra & $0,48 \%$ & $0,11 \%$ \\
\hline Baleares & $0,57 \%$ & $0,09 \%$ \\
\hline Comunidad Valenciana & $0,46 \%$ & $0,01 \%$ \\
\hline Castilla La Mancha & $0,45 \%$ & $0,01 \%$ \\
\hline País Vasco & $0,41 \%$ & $0,33 \%$ \\
\hline La Rioja & $0,31 \%$ & $0,05 \%$ \\
\hline Andalucía & $0,30 \%$ & $0,14 \%$ \\
\hline Asturias & $0,30 \%$ & $0,10 \%$ \\
\hline Canarias & $0,24 \%$ & $0,01 \%$ \\
\hline Cataluña & $0,22 \%$ & $0,05 \%$ \\
\hline Cantabria & $0,21 \%$ & $0,01 \%$ \\
\hline Madrid & $0,21 \%$ & $0,00 \%$ \\
\hline Aragón & $0,17 \%$ & $0,04 \%$ \\
\hline Extremadura & $0,16 \%$ & $0,16 \%$ \\
\hline Murcia & $0,11 \%$ & $0,00 \%$ \\
\hline Castilla y León & $0,10 \%$ & $0,04 \%$ \\
\hline Galicia & $0,9 \%$ & $0,5 \%$ \\
\hline
\end{tabular}

Fuente: Intermón Oxfam (2015)

http://www.realidadayuda.org/analizar-la-ayuda/ayuda-autonomica

Los diferentes informes de la entidad hacen visible y evidente que nunca y en ninguna Comunidad Autónoma se ha alcanzado el simbólico 0,7\% que se reclamó en las famosas acampadas iniciadas en el Paseo de la Castellana de Madrid en 1994. Un clamor de solidaridad y reivindicación que se extendió por todo el estado español, y que sirvió para traducir el compromiso en el objetivo de alcanzar el $0,7 \%$ del PIB para luchar contra la pobreza y reducir las desigualdades del Planeta. Una acción que Víctor Marí (2013) enmarca en un periodo de eclosión y que significó la puesta de largo de las organizaciones y de la ciudadanía comprometida con la solidaridad y los países del sur empobrecidos. Igualmente los datos de Oxfam demuestran comunidades, como son la del País Vasco y Andalucía, que a pesar de recortar, se han mantenido entre los seis primeros puestos del ranking (excepto en el 2011 en las que Andalucía se sitúa en el puesto 14 de las 17 comunidades) de la horquilla seleccionada. De hecho es el País Vasco en el 2012 con un 0,52\% quien ha estado más cerca de la simbólica cifra. Por otro lado, los diferentes informes también muestran la otra cara de la moneda demostrando las caídas brutales de casos como el de la Comunidad de Valencia que del $0,46 \%$ en 2008 , se quedó en un $0,01 \%$ en 2015 . O el de las comu- 
nidades de Madrid y Murcia que directamente en el mismo año dejan de financiar la cooperación internacional al desarrollo. En el caso de Andalucía, los recortes a las políticas públicas de cooperación no fueron tan drásticos en relación a las comunidades anteriormente señaladas. Este hecho podría estar justificado por el compromiso político y el respaldo social reflejado en el artículo 10.3 del Estatuto de Autonomía que indica: "La cooperación internacional con el objetivo de contribuir al desarrollo solidario de los pueblos". E incluso en el Pacto Andaluz por la Solidaridad y la Cooperación Internacional ${ }^{4}$.Un documento en el que se pone de manifiesto que la pobreza no es una cuestión exclusiva de los países pobres y que su solución requiere del esfuerzo de todos.

Los datos recogidos en la tabla, a partir del informe anteriormente citado, posicionan a Andalucía en el séptimo puesto de las comunidades autonómicas con un $0,30 \%$ en 2008 , para pasar a la segunda posición con un $0,14 \%$ en 2015 . Lo significativo es que Andalucía gana posiciones con respecto al resto de comunidades en un año que va recuperando la caída del 2011, pero en el que sigue siendo una aportación inferior a la de principios de la crisis.

Tabla 2. Aportaciones de la Comunidad de Andalucía.

\begin{tabular}{|c|c|c|c|c|c|c|c|c|}
\hline Año & $\mathbf{2 0 0 8}$ & $\mathbf{2 0 0 9}$ & $\mathbf{2 0 1 0}$ & $\mathbf{2 0 1 1}$ & $\mathbf{2 0 1 2}$ & $\mathbf{2 0 1 3}$ & $\mathbf{2 0 1 4}$ & $\mathbf{2 0 1 5}$ \\
\hline$\%$ & $0,30 \%$ & $0,29 \%$ & $0,24 \%$ & $0,05 \%$ & $0,10 \%$ & $0,15 \%$ & $0,10 \%$ & $0,14 \%$ \\
\hline
\end{tabular}

Fuente: Intermón Oxfam (2015)

http://www.realidadayuda.org/analizar-la-ayuda/ayuda-autonomica

En definitiva, como ya adelantaban Martínez y Martínez (2013), el sector de la cooperación española, y en el que hay que incluir a Andalucía, en su conjunto se ve afectado en cinco puntos:

1. Ruptura del consenso sobre el crecimiento de la política, con un desplome de los recursos.

2. Ruptura del consenso sobre el objetivo de la política.

3. Ruptura del consenso sobre el fortalecimiento institucional.

4. Ruptura del consenso sobre el desarrollo.

5. Ruptura del consenso social.

Esta investigación se ha centrado en analizar la reacción comunicativa del Grupo de Comunicación de la $\mathrm{CAONGD}^{5}$, especialmente a partir de la campaña conmemorativa de los '20 años del $0,7 \%$. Ya que la estrategia perseguía, entre otras cosas, recortar, en la medida de lo posible, el distanciamiento de una parte de la sociedad (ruptura del consenso social) con la cooperación internacional al desarrollo.

$4 \quad$ Firmado por el entonces vicepresidente de la Junta de Andalucía, Diego Valderas, el 23 de junio de 2013 junto a un centenar de ONGD, agencias de Naciones Unidas, universidades andaluzas y otros actores.

5 La Coordinadora Andaluzas de ONGD (CAONGD) es una plataforma que actualmente aglutina a 66 organizaciones de desarrollo y seis plataformas provinciales. La CAONGD se crea en 1993 y jugará un papel decisivo en las reivindicaciones del 0,7\% del año 1994. 
González Álvarez (2010) bajo su definición de una comunicación para la solidaridad defiende un modelo encaminado a despertar la solidaridad en las personas y las sociedades para lograr modificar la realidad de forma positiva. Balas Lara (2008) insistía en que la confianza social es la base y justificación de las organizaciones sociales. Por lo tanto tiene sentido preguntarse ¿qué puede hacer la comunicación para recuperar el consenso social en un escenario de crisis?

Raigada dice que (1997) todas las crisis comparten una misma característica: no existe ninguna crisis totalmente anticipada. Es decir, si se conociera previamente, seguramente no hubiera dado lugar a la denominada crisis. Por lo que cada crisis es única. Sin embargo Ana Enrique (2008) afirma que se dan dos fases: una situación de pre-crisis, en la que se detectan los primeros riesgos. Momento en los que habría que hacer una primera evaluación, y si es posible, tomar medidas en torno a un plan de comunicación que debería frenar o contrarrestar las consecuencias de lo que se avecina; y la crisis, que una vez encima hay que asumirla y aceptarla para abordarla con claridad comunicativa.

Por lo tanto, asumida la crisis, la respuesta comunicativa de las entidades se podían desarrollar en el escenario tradicional que como explica Barranquero (2014) tienen rasgos aún no superados de estrategias pensadas casi exclusivamente en atraer y dar respuesta a la demanda de los medios de comunicación (mediocentrismo); condicionadas por los acontecimientos y sin planificación (cortoplacismo); y con acciones jerárquicas, alineadas con el marketing social, y pensadas para vender una entidad, una causa o una marca (mercadeo).O bien, como han venido haciendo, en ocasiones, la propia CAONGD en anteriores campañas (un ejemplo de ello es la que se llamó 'Cocina otra mundo' ${ }^{\text {}}$ ) alineadas con los rasgos de la comunicación para el cambio social. Un modelo teorizado entre otros por Gumucio $(2004,2011)$ y Víctor Marí $(2011,2013)$ que sitúan a la comunicación como parte de un proceso, abierto a la participación de nuevos actores y medios, a mensajes que van a explicar las causas y las consecuencias, a empoderar a la ciudadanía...para comprometerlos como parte activa y necesaria de la transformación social.

\section{Metodología}

Para entender la forma de reacción de la CAONGD además de la observación constante de los acontecimientos y el seguimiento a sus acciones comunicativas, se realiza una entrevista ${ }^{7}$ semiestructurada a Maribel Roldán, Lourdes Rodríguez y Hazeina Rodríguez. Ya que las tres forman parte del núcleo principal del Grupo de Comunicación integrado en la Coordinadora y además juegan un papel decisivo en el desarrollo de la campaña conmemorativa de los '20 años del $0,7 \%$ '.

El análisis de la investigación es de corte cualitativa. Ya que este modelo responde a cierta sensibilidad social (Íñiguez, 1999). Y se opta por la realización de una entrevista, de carácter semiestructurada, ya que esta herramienta permite el contacto directo con el individuo (entrevistado) protagonista del objeto de estudio (Taylor \& Bogdan, 1987). La entrevista brinda la construcción de las conclusiones a partir de

'Cocina otro mundo' (2012) fue una campaña colectiva realizada de forma participativa en formato videoblog por el Grupo de Comunicación de la CAONGD destinadas a acercar a la ciudadanía al mundo de la cooperación como instrumento para luchar contra la pobreza. En la acción audiovisual participaron más de 50 personas de casi 60 de organizaciones.

7 La entrevista se realizó el 20 de septiembre del 2016 en la sede de la CAONGD en Sevilla. 
las opiniones y conexión con los entrevistados, teniendo muy en cuenta el contexto en el que se produce.

Las preguntas formuladas fueron las que se recogen en la siguiente tabla.

Tabla 3. Cuestionario entrevistas.

\begin{tabular}{|l|l|}
\hline \multicolumn{1}{|c|}{ Fuente Bloques } & \multicolumn{1}{c|}{ Preguntas } \\
\hline $\begin{array}{l}\text { Cooperación al } \\
\text { desarrollo }\end{array}$ & $\begin{array}{l}\text { Se ha pasado uno de los periodos de mayor recorte en el ámbito de la } \\
\text { cooperación al desarrollo, ¿cómo ha afectado al mundo de las ONGD } \\
\text { andaluza? } \\
\text { ¿Cuál ha sido la estrategia de comunicación realizada? ¿Ha tenido éxito? } \\
\text { ¿Qué papel ha jugado la cooperación ante la situación de crisis/recortes en } \\
\text { el ámbito de la cooperación? }\end{array}$ \\
\hline $\begin{array}{l}\text { Campaña '20 años } \\
\text { del 0,7\%' }\end{array}$ & $\begin{array}{l}\text { ¿Por qué surge? } \\
\text { ¿Cómo se estructura? } \\
\text { ¿Ha cumplido con sus objetivos? } \\
\text { ¿Qué repercusión ha tenido en los medios? ¿Y entre otros actores: sociedad, } \\
\text { administraciones etc...? }\end{array}$ \\
\hline
\end{tabular}

Fuente: Elaboración propia.

El cuestionario fue revisado con anterioridad por la periodista responsable de Comunicación del Fondo Andaluz de Municipios por la Solidaridad (FAMSI) quien manifestó:

“Creo que el tema es muy interesante, no tanto por la campaña en sí misma, sino por los cambios que impulsó la movilización en su momento ..."

"Cuando leía el cuestionario pensaba que esta campaña de los 20 años la vi interesante porque era como revitalizar aquella indignación, por supuesto, sensibilizar y reivindicar, pero también hacer incidencia política en un momento muy clave."

Igualmente se estudian los materiales comunicativos presentados para la campaña.

Tabla 4. Materiales y objetivos de la campaña.

\begin{tabular}{|l|l|}
\hline \multicolumn{1}{|c|}{ Materiales } & \multicolumn{1}{c|}{ Objetivos } \\
\hline $\begin{array}{l}\text { Audiovisuales } \\
\text { Documental (versión reducida de 12 minutos). Disponible } \\
\text { en: https://www.youtube.com/watch?v=omBBjypx__4 } \\
\begin{array}{l}\text { Documental completo (50 minutos). Disponible en: } \\
\text { https://www.youtube.com/watch?v=AxAe7fRgPzA }\end{array}\end{array}$ & $\begin{array}{l}\text { Recuperar la memoria colectiva } \\
\text { a través de la reflexión de los } \\
\text { protagonistas en las acampadas en } \\
\text { las capitales andaluzas. }\end{array}$ \\
\hline $\begin{array}{l}\text { Exposición } \\
13 \text { enaras de un metro de ancho por dos de alto }\end{array}$ & $\begin{array}{l}\text { Servir para reivindicar la } \\
\text { importancia de la cooperación } \\
\text { internacional. }\end{array}$ \\
\hline
\end{tabular}

Fuente: Elaboración propia. 
Tras las entrevistas, se celebra un grupo de discusión ${ }^{8}$ formado por dos profesionales de los medios de comunicación, dos expertos en movimientos sociales, una experta en cooperación internacional y dos académicas del campo de la Economía y la Sociología especializadas en desarrollo y movimientos sociales. Este grupo busca reflexionar sobre si la comunicación (y la información como parte de ella) contribuye al cambio social.

Se opta por esta técnica porque como sostiene Hernando y Montilla Coronado (2009) facilita la aparición del discurso, el contraste de opiniones y las posiciones de los participantes.

La confrontación de las entrevistas y del grupo de discusión brindan los siguientes resultados.

\section{Resultados}

Todo hace indicar que el sector de la cooperación con sello andaluz no vio venir la crisis. Ni tuvo un plan de comunicación específico para abordar lo que se venía encima. Más bien reaccionó, en una primera fase, con una estrategia de comunicación pensada en conectar con los medios (generalistas y alternativos) a través de mensajes centrados en la denuncia de los recortes. Un modelo de estrategia mediocentrista -como apuntaba Barranquero- e instrumental centrada, principalmente, en lograr la atención de los medios, en vez de una noción de lo comunicativo que logre dar cuenta de toda la dimensión comunicativa (Erro, 2002, 2003,2004, 2012). Es decir, acciones encaminadas a ocupar espacios mediáticos basados en informar sobre las consecuencias directas de pérdida de recursos económicos en las organizaciones y no tanto en las causas globales -en materia de justicia social, de compromiso exterior, de acuerdos internacionales-. Además, estos mensajes se dieron de bruces con el aluvión de otros colectivos locales que, igualmente, denunciaban las consecuencias de los recortes en el terreno de la salud, de la educación, o del empleo...y que conectaban mejor con la ciudadanía y despertaban mayor interés en los medios de comunicación.

Maribel Roldán (MR1): “Actuamos comunicativamente más por supervivencia, pero sin una estrategia definida".

Al principio, las acciones comunicativas se centran en insistir, una y otra vez, sobre los recortes de forma general y sus consecuencias en el sector, especialmente en las organizaciones. La estrategia comunicativa se centra en la denuncia. Los resultados no son los esperados. Hay una enorme competencia mediática. El interés de los medios está en las Mareas ciudadanas que ponen el foco en los recortes en sanidad, educación, empleo etc. Además, comparativamente con otras comunidades, Andalucía está manteniendo la ayuda. El recorte, proporcionalmente, es mínimo. El problema está en el retraso de las ayudas concedidas.

MR2: Y un día me harté. No mando ninguna nota de prensa con lo mismo: diciendo que nos deben, que la Junta (de Andalucía) tiene tantos millones de retraso...

8 El grupo de celebró el 26 de junio de 2017 en la sede de Radio Hispanidad (Huelva). 
Eso no generaba ninguna corriente a favor del trabajo de las organizaciones. Era uno más. Y además, en un momento que se instauró el mensaje de los de aqui antes de los de fuera".

La puesta en marcha de la campaña '20 años del 0,7\%' brinda a las entidades andaluzas dar un giro en su estrategia comunicativa. En buena medida, ampliando la dimensión comunicativa y recuperando el sentir de Freire de entender la comunicación como instrumento de concienciación, o en la de Bordenave (2013) como un instrumento para construir otro mundo posible por medio de otra comunicación posible. Este nuevo posicionamiento hace que la campaña sea una nueva oportunidad comunicativa para encarar los efectos de la crisis y recuperar a la ciudadanía. En la línea apuntada por la experta al referirse a los objetivos del cambio social "reclaman discursos construidos desde y hacia las emociones movilizadoras y creencias en la posibilidad de cambio definido como la transformación de las injusticias que son enmarcadas como problema colectivo fruto de unas determinadas políticas y acciones" (Nos Aldás, 2015:5).

La campaña es una propuesta de sensibilización, información y reivindicación, que contó con financiación de la Agencia Andaluza de Cooperación Internacional para el Desarrollo (AACID), y que aprovecha, en términos periodísticos, la percha conmemorativa de la acción colectiva gráficamente representadas en las acampadas por todo el territorio nacional y en todas las capitales de las ocho provincias andaluzas que reivindicó el 0,7\% y situó en la agenda política y social la cooperación internacional. Para algunos, como Jerez y Sampedro (2004) además sirvió para despertar el interés mediático al lograr una imagen pública del sector de la cooperación y lograr un discurso de consenso.

La campaña se estructura a través de un documental y una exposición que recupera imágenes, noticias y protagonistas de las acampadas que ocuparon las plazas de las ciudades andaluzas. El audiovisual, dirigido por Hazeina Rodríguez, recoge el testimonio de 16 activistas que se movilizaron en el otoño de 1994 en Cádiz, Córdoba, Granada, Huelva, Jerez, Málaga y Sevilla. En sus cuatro bloques se abordan los orígenes y el desarrollo de las acampadas; la repercusión social, mediática y política de este movimiento; sus conexiones con otras reivindicaciones posteriores, como el 15M; así como la situación actual que vive la cooperación internacional al desarrollo.

En seis de estas ciudades se organizó un acto de proyección, seguido de un foro de debate protagonizado por algunos de los protagonistas locales de las acampadas sobre la situación actual de la cooperación internacional en Andalucía y que además sirvió para rememorar la lucha colectiva a favor de este instrumento. La exposición estuvo en bibliotecas, centros universitarios, estaciones de tren o delegaciones territoriales de Canal Sur, entre otros contó con la colaboración de RENFE y la televisión pública andaluza (RTVA). La campaña se estrenó el 4 de diciembre de 2014 y finalizó en mayo de 2015. Los organizadores dan una estimación de público que ronda las 20.000 personas.

Sin embargo, las entrevistadas reconocen no tener indicadores objetivos y cuantificables para medir el impacto real entre la ciudadanía. Si reconocen cierto éxito en la estrategia de recuperar la memoria colectiva, y de contribuir a reconstruir y a unificar el discurso a favor de la cooperación internacional.

Las entrevistadas, por un lado, critican la poca repercusión mediática, especialmente en medios generalistas. Igual una de las causas se encuentran en estas palabras 
"los medios acostumbran a convertir en noticia asuntos de enorme banalidad y en banalizar la información trascendente. El debate real se hurta porque interesa más vender la espectacularidad de la noticia. Son discursos tan contradictorios que no permiten a la ciudadanía hacer esa necesaria construcción de la realidad que nos situaría en la antesala de una opinión crítica y constructiva, implicada en las transformaciones necesarias" (Chaparro, 2012:28).

Y por otro lado vuelven a reconocer el déficit que tienen como estructura al no medir sus acciones.

MR3: "Es complicado (dar un dato). No medimos el impacto. No medimos el impacto de las acciones de comunicación de las organizaciones. Es un déficit que tenemos".

Lourdes Rodríguez (LR1): "Hacemos cuánta gente vista la web...y esas cosas... pero eso, no mide el impacto".

Las promotoras de la iniciativa sí que consideran que la campaña les brindó nuevas oportunidades para reconducir la estrategia a una comunicación pensada en las claves para el cambio social: generando más participación a partir de una mejor articulación de redes y alianzas; optimizando esfuerzos y uniendo una sola voz en un mensaje claro contra los recortes fuera en el ámbito que fuera; recuperando la memoria colectiva como instrumento para reivindicar que la cooperación, veinte años después, seguía siendo una cuestión de justicia social y de compromiso con la posibilidad real de construir un mundo más justo; dando voz y visibilidad a otras voces y protagonistas.

MR4: "La campaña del $0,7 \%$ era renovar la movilización social a favor de esta historia. Y si en ese momento estaba justificada, hoy seguía estando. Pasamos de comunicar que nos estamos yendo al traste por los recortes, a comunicar de otra manera, a seguir diciendo que el trabajo era necesario".

LR2: "También vi un giro en agrupar con otros movimientos. Hasta ahora, como todo el mundo, cada uno había estado defendiendo su parcelita. Y creo que se da un giro...Y tener toda una visión de conjunto. Se avanzó en la línea de buscar los puntos en común".

Mientras en el Grupo de Discusión los participantes coinciden en reconocer el papel de la comunicación e información como instrumentos y parte del proceso capaz de generar cambios en la sociedad. E incluso hay una doble reivindicación: una encaminada a insistir que debe estar en el ADN de toda entidad el hacer comunicación como parte activa del cambio y como vehículo para mantener la imprescindible conexión entre la ciudadanía y las ONGD; y la segunda, en reclamar a los medios de comunicación y especialmente a los periodistas para que recuperen su rol de servicio público y, por tanto, sean aliados y partícipes en la difusión de las acciones y objetivos de las ONGD.

Experto 1: "Entre la hiperinformación y la turbidez mediática es importante la profesionalización de la información para ponerla al servicio de la ciudadanía".

Experto 2: "Partiendo de la diferencia entre comunicación e información insisto en la necesidad que los medios de comunicación tienen que ser medios de servicios públicos y ejercer su función social". 
Experto 3: "La comunicación forma parte de la sociedad, al ser el canal por el que nos contamos las cosas, por el que articulamos el pensamiento".

\section{Conclusiones}

Hay que partir del hecho que la crisis financiera internacional ha supuesto un claro retroceso en la AOD, un alejamiento del objetivo de alcanzar el 0,7\% del PIB en materia de cooperación internacional y un distanciamiento de España como actor de peso en el panorama internacional. Igualmente se ha evidenciado que las comunidades autónomas han reaccionado de manera dispar e incluso absolutamente antagónicas como son desde la eliminación total del presupuesto de las comunidades de Madrid o Murcia, al tibio aumento en Andalucía o el algo superior del País Vasco. Detrás de estas decisiones hay claros posicionamientos ideológicos.

Este contexto descrito de recortes y consecuencias en el ámbito de la cooperación y de sus actores, seguramente, requería de planes de comunicación para una situación de crisis si las organizaciones no querían perder su espacio, su peso y especialmente su conexión con la ciudadanía. Una ciudadanía que como recuerdan Cascante y Rodríguez (2017), a pesar de mantener un aparente discurso contradictorio, sigue manifestando en las encuestas el convencimiento que la AOD es necesaria y mostrando su confianza en el papel de las ONGD. Sin embargo se reaccionó mal y tarde. Las prisas, la falta de previsión y de escasos recursos en las áreas de comunicación hizo que se volviera a las acciones comunicativas convencionales pensadas en ocupar espacio y tiempo en los medios de comunicación sin medir este (en relación al espacio y al tiempo) ya estaba siendo ocupado por otros colectivos víctimas de los recortes en los pilares del estado del bienestar.

Las entrevistadas reconocen una reacción tardía e incluso unas medidas comunicativas, en una primera fase, muy centradas en reivindicaciones generales que no conectaban con el sentir mayoritario de la sociedad, ni con el interés de los medios de comunicación (entre otros motivos, por una saturación y por un mayor interés en las consecuencias directas de los recortes en ámbitos como los sanitarios, sociales y educativos). La puesta en marcha de la campaña '20 años del $0.7 \%$ ' sirvió gancho para reconducir la estrategia comunicativa hacia mensajes más centrados en lo emocional (en la línea apuntada por Aldás) -un llamamiento a la memoria colectiva a través de dar visibilidad y reconocimiento a las personas que participaron con sus denuncias y peticiones en las acampadas históricas que sirvieron para demandar el $0,7 \%$ del PIB en cooperación y posicionar al sector de las ONGD; evidenciar que los cambios son posibles desde el empoderamiento y desde la participación en causas colectivas; con mensajes positivos encaminados a explicar el impacto que tiene la cooperación al desarrollo en el bienestar de las personas, en la lucha contra la pobreza y en la reducción de las desigualdades. Todo ello enmarcado en una estrategia conmemorativa que sirvió para el reencuentro de activistas, para evidenciar que el objetivo propuesto hace 20 años seguía sin alcanzarse, para sumar voces y acciones que insistían de forma unida que los recortes eran injustos, ideológicos, impuestos y que afectaban a los derechos universales. Igualmente es destacable que la campaña posibilitó la transversalidad y las alianzas. Ya no se trataba por competir por el espacio y el tiempo mediático, ni tan poco por la conectividad con la ciudadanía. Si no en sumar esfuerzos y evidenciar que la crisis y sus 
efectos son decisiones políticas con consecuencias que afectan de forma general a las personas.

Parece más que evidente la importancia de la comunicación para las organizaciones. La comunicación sigue siendo el medio de conexión con la sociedad. Clave en las acciones de sensibilización, denuncia, incidencia política y transformación social. Se hace visible la necesaria relación entre los actores de la comunicación y los medios (generalistas o especializados, locales o nacionales, convencionales o alternativos) para la difusión de mensajes y para conectar con la ciudadanía. Una vez más, compartiendo lo expresado por los participantes del grupo de discusión, la comunicación se perfila como un instrumento vital para los procesos de transformación y cambio social. Las entidades necesitan definitivamente poner en valor este instrumento, dotando a las estructuras de los recursos necesarios, para responder a las nuevas demandas informativas y comunicativas de una sociedad hiperconectada e hipersaturada que atenderá a los mensajes si estos aportan contenidos de calidad, especializados y son atractivos en su forma de ser presentados. Todo ello sin caer en el canto de sirena del envoltorio, en la estrategia centrada en los canales que, en demasiadas ocasiones, hace olvidar que los instrumentos están al servicio de la comunicación y no a la inversa. Es imprescindible que las ONGD no pierdan su rumbo, que no puede ser otro que lograr un mundo más justo. Para ello, se hace necesario un modelo de comunicación más participativo, crítico, educativo y transformador, es decir, una verdadera comunicación al servicio del cambio social.

Las limitaciones principales se encuentran, por un lado, en las propias organizaciones que siguen respondiendo de forma autómata con modelos comunicativos convencionales. Falta una interiorización y una práctica real y continuada de modelos comunicativos en la línea de la comunicación para el cambio social. Siguen faltando datos cuantitativos que midan con objetividad los resultados de sus estrategias de comunicación. La CAONGD requeriría de más recursos para que el departamento y el Grupo de Comunicación (que cuenta con profesionales cualificados y convencidos en que otro mundo posible, requiere de otra comunicación posible) pudieran elaborar, ejecutar, evaluar y sistematizar un plan de comunicación para el y por el cambio social.

Sería recomendable, como sugieren en el grupo de discusión, que las ONGD no olvidarán el fin de su trabajo, y que la comunicación de dichas ONGD estuviese a cargo de profesionales de éste área. Es fundamental construir la comunicación con la ciudadanía y para la ciudadanía. Si la identidad de las organizaciones "lleva implícita una concepción de la comunicación basada en el establecimiento de un proceso de diálogo capaz de interpelar a los ciudadanos y ciudadanas a nivel individual a pesar de que los canales sean colectivos" (Lucerga, 2013:85), seguramente hay que hacer un mayor esfuerzo comunicativo. Ya que el respaldo ciudadano, que se logra con una ciudadanía formada y crítica, da sentido al trabajo de las organizaciones por lograr sociedades justas, igualitarias y sostenibles, y garantizan como dique de contención ante los discursos reaccionarios que intentan que la cooperación internacional no esté en la agenda política de las comunidades autónomas.

Finalmente, es precisamente esta nueva ciudadanía global la que brinda, por un lado, nuevas posibilidades de estudio para diseñar nuevas estratégicas comunicativas e informativas que no pierdan el objetivo transformador pero que incluyan nuevos formatos discursivos y nuevos espacios de interrelación para que el compromiso con la cooperación internacional se entienda como un derecho y no una práctica sujeta decisiones económicas y políticas. 


\section{Referencias bibliográficas}

Balas, L. M (2008) El reto de la comunicación en el sector no lucrativo. Revista Española del Tercer Sector. № 8 . Madrid.

Cascante, K \& Rodríguez E. (2014). El impacto de la crisis sobre el tejido social solidario en España: efectos y reacción de las ONGD frente a la crisis. Laboratorio de Alternativas. Fundación Iniciativas por la Paz. Madrid.

Chaparro, E. M. (2012). Medios de comunicación y democracia. Lecciones desde América Latina. Commons: revista de comunicación y ciudadanía digital, 1(1), 15-34.

Barranquero, A. (2014). Comunicación, cambio social y ONG en España. Pistas para profundizar en la cultura de la cooperación desde los nuevos movimientos comunicacionales.El caso del 15M. Commons: Revista de Comunicación y Ciudadanía Digital, V.3, N.), pp. 6-28. Recuperado http://hdl.handle.net/10016/20699

CONGDE (2009), Informe sobre el sector de las ONGD 2008. Accesible en www.congde. org. Recuperado 18/05/2017.

CONGDE (2011), Informe sobre el sector de las ONGD 2010. Accesible en www.congde. org. Recuperado 18/05/2017

CONGDE (2012), Informe sobre el sector de las ONGD 2011. Accesible en www.congde. org. Recuperado 18/05/2017.

CONGDE (2013), Informe sobre el sector de las ONGD 2014. Accesible en www.congde. org. Recuperado 18/05/2017.

Díaz Bordenave, J. (2013). La comunicación y el nuevo mundo posible. Commons. Revista de Comunicación y Ciudadanía Digital, 1(6-12).

Enrique. A.M (2008) La gestión de la comunicación en situaciones de crisis. El caso de la compañía United Biscuits en la crisis de Fontaneda. Comunicación y Sociedad. Vol. XXI - Núm. 2 (37), 35-56.

Erro.S, J, (2012): Comunicación, cooperación internacional para el desarrollo y ONGD: un modelo de trabajo desde la educación y la cultura". En Burgui, T y Errol, J (Coords.), Comunicando para la solidaridad y la cooperación. Cómo salir de la encrucijada.

Erro.S, J. (2003) II ONGD: ¿Comunicarse porqué y para qué? El paso de la comunicación mercadeada a la comunicación social educativa. En Benet V, J y Nos. A. E (Coords) La publicidad en el tercer sector: tendencias y perspectivas de la comunicación solidaria (32, 53). Barcelona, Icaria.

Erro. S. J (2004): « ¿Comunicar en valores? Una propuesta educadora para las ONGD», en V.Marí Sáez (Coord.), La red es de todos. Cuando los movimientos sociales se apropian de la red, E. Popular, Madrid, páginas 116-134.

Fanjul, G (15 diciembre 2015) Las tres derrotas de la cooperación española. El País. http://elpais. com/elpais/2015/12/09/planeta_futuro/1449654250_213294.html. Recuperado 24/02/2018

González, A, M.I (2012) La profesionalización de la Comunicación para la Solidaridad: diagnóstico y propuestas para el sector en España.CIC, Cuadernos de Información y Comunicación. Volumen 17 pp 242-243.

Gumucio Dagron, A. (2001). Haciendo olas: historias de comunicación participativa para el cambio social. New York. Estados Unidos de América: The Rockefeller Foundation.

Gumucio-Dagron, A. (2004). El Cuarto Mosquetero: La comunicación para el cambio social. Investigación y Desarrollo volumen 12, nº1. 2-7. Recuperado de goo.gl/kqrsQ3

Hernando, Á. y Montilla, Ma . (2009). El estudio de los roles y funciones de los orientadores de secundaria utilizando la técnica de los grupos de discusión. Revista Española de Orientación y Psicopedagogía, 20 (1), 29-38. 
Intermón Oxfam (2013). Informe Realidad de la Ayuda. Accesible http://www.realidadayuda.org/.

Lucerga, M.J. (2013). Nuevas herramientas para viejos interrogantes. Contradicciones de la comunicación de las organizaciones de desarrollo en la era de la solidaridad tuiteada. Commons. Revista de Comunicación y Ciudadanía Digital, 3(85) 66-86.

Marí-Sáez, V.M. (2010). El enfoque de la comunicación participativa para el desarrollo y su puesta en práctica en los medios comunitarios. Razón y Palabra, 71, 40.

Marí-Sáez, V.M. (2011): Comunicar para transformar, transformar para comunicar. Tecnologías de la información desde una perspectiva de cambio social. Madrid. Editorial Popular.

Mari S. Víctor (2013) Comunicación, desarrollo y cambio social en España: entre la institucionalización y la implosión del campo. Revista Commons $\mathrm{n}^{\mathrm{o}} 3$.

Mari Sáez, Victor (2013) Comunicación, desarrollo y cambio social en España: entre la institucionalización y la implosión del campo. Revista Commons no 3. 42-64.

Martínez, I \& Martínez, P. (2010) Plataforma 2015 y más. Recuperado de goo.gl/GkBlLp. (Consultado 10 de junio 2017).

Martínez, P. y Martínez, I. (2014). Ruptura del consenso y desmantelamiento: un análisis de la cooperación española. Anuario 2013. Hacia 2015: Visiones del desarrollo en disputa. XI. Informe Anual de la Plataforma 2015 y más. Recuperado de http://www.2015ymas. org/.

Jerez, A \& Sampedro, V (2004) Visibilidad pública y tratamiento informativo del movimiento de cooperación al desarrollo (1992-2002). Política y Sociedad. 50-53.

Raigada, J. L. (1997). La comunicación corporativa de la gestión de crisis. Teoría de La comunicación y gestión de las organizaciones. Madrid: Síntesis.

Sotillo, J. Á. (2014). La política exterior y la política de cooperación: paradojas del caso español. Comillas Journal of International Relations, (1), 117-131.

Taylor, S. J., \& Bogdan, R. (1987). Introducción a los métodos cualitativos de investigación (Vol. 1). Barcelona: Paidós. 